\title{
Terapia Hormonal na Menopausa: Quando Não Usar
}

\section{mini-revisão}

\section{Poll MARA SPRITZER Maria Celeste Osório Wender}

Unidade de Endocrinologia Ginecológica, Serviço de Endocrinologia (PMS), e Setor de Menopausa, Serviço de Ginecologia e Obstetrícia (MCOW), Hospital de Clínicas de Porto Alegre, Universidade Federal do Rio Grande do Sul, Porto Alegre, RS.

Recebido em 04/06/07 Aceito em 02/07/07

\section{RESUMO}

A menopausa corresponde à cessação permanente da menstruação, conseqüente à perda da função folicular ovariana ou à remoção cirúrgica dos ovários. A idade média para ocorrência da menopausa natural gira em torno de 50 anos. A deficiência estrogênica decorrente da menopausa está associada com sintomas vasomotores, atrofia urogenital, declínio cognitivo, assim como a um aumento no risco de doenças crônico-degenerativas, aterosclerose e doença cardiovascular, osteoporose e doença de Alzheimer. A estrogenioterapia permanece sendo o tratamento mais efetivo para o manejo dos sintomas vasomotores e atrofia urogenital. Em mulheres com útero presente, a progesterona natural ou os progestogênios devem ser associados ao tratamento com estradiol para antagonizar os efeitos proliferativos deste hormônio sobre o endométrio e anular o risco de hiperplasia/carcinoma endometrial. Por outro lado, em determinadas condições clínicas, a terapia hormonal não é recomendada ou é mesmo contra-indicada. Neste artigo, focalizamos criticamente essas situações clínicas em que não se deve indicar a terapia hormonal na menopausa. (Arq Bras Endocrinol Metab 2007;51/7:1058-1063)

Descritores: Estrogênios; Progestogênios; Menopausa; Carcinoma de mama; Tromboembolismo; Sangramento vaginal

\section{ABSTRACT}

Hormone Therapy in Menopause: When Not to Use.

Menopause is defined as the permanent cessation of menses, as a result of the loss of ovarian follicular function or of surgical removal of ovaries. The mean age for occurrence of natural menopause is around 50 years. Estrogen deficiency has been associated with vasomotor symptoms, urogenital atrophy, and cognitive impairment, as well as increased risk of chronic degenerative diseases such as osteoporosis and Alzheimer's disease. Estrogen therapy remains the most effective treatment for the management of vasomotor symptoms and urogenital atrophy. Progesterone or progestins should be added to estrogen treatment in women with uterus, in order to antagonize the estrogen-induced endometrial proliferation. In turn, in specific clinical conditions hormone therapy is not recommended. In the present article, the authors critically focus these clinical conditions in which hormone therapy should not be used. (Arq Bras Endocrinol Metab 2007;51/7:1058-1063)

Keywords: Estrogens; Progestins; Menopause; Breast cancer; Tromboembolism; Vaginal bleeding 
A MENOPAUSA É DEFINIDA PELA Organização Mundial da Saúde como a cessação permanente da menstruação, conseqüente à perda da função folicular ovariana ou à retirada cirúrgica dos ovários (1). A idade média para ocorrência da menopausa natural gira em torno de 50 anos $(1,2)$.

As mudanças hormonais que acompanham a menopausa, principalmente a diminuição nos níveis de estrogênio, têm grande impacto fisiológico. A deficiência estrogênica tem sido implicada com sintomas vasomotores, atrofia urogenital, declínio cognitivo, assim como a um aumento no risco de doenças crônico-degenerativas, como a aterosclerose e doença cardiovascular, osteoporose e doença de Alzheimer.

Desde as últimas décadas, o manejo da paciente menopáusica representa tema de grande interesse na área clínica, epidemiológica e de saúde pública, devido, em grande parte, à melhora na expectativa de vida, que permite prever que a população feminina possa viver cerca de um terço de suas vidas após a menopausa. Estudos demográficos indicam que em 1990 havia cerca de 467 milhões de mulheres com idade igual ou superior a 50 anos. Para o ano de 2030, a estimativa é que este grupo ultrapasse 1.200 milhões de mulheres (3).

Mais recentemente, contudo, e em conseqüência à publicação de ensaios clínicos randomizados envolvendo um grande número de mulheres, a terapia hormonal na menopausa tornou-se uma questão complexa, que vem gerando um intenso debate entre especialistas e uma expressiva divulgação na mídia leiga, nem sempre acompanhada da necessária reflexão e ponderação sobre as evidências científicas, os limites e as perspectivas originadas por estes trabalhos.

A estrogenioterapia permanece sendo o tratamento mais efetivo para o manejo dos sintomas vasomotores e atrofia urogenital (4). Em mulheres com útero presente, a progesterona natural ou os progestogênios devem ser associados ao tratamento com estradiol para antagonizar os efeitos proliferativos deste hormônio sobre o endométrio e anular o risco de hiperplasia/carcinoma endometrial (5-9). Embora no momento atual não representem a indicação primária para a terapia hormonal em mulheres pós-menopáusicas assintomáticas, o uso de estradiol é também reconhecido no tratamento da osteoporose pós-menopáusica $(10,11)$ e pode diminuir a incidência de carcinoma de cólon (12).

Por outro lado, em determinadas condições clínicas, a terapia hormonal não é recomendada ou é mesmo contra-indicada. Neste artigo, focalizamos criticamente essas situações clínicas em que não se deve indicar a terapia hormonal na menopausa.

\section{CARCINOMA DE MAMA}

No momento atual, a terapia hormonal é contra-indicada após um diagnóstico de câncer de mama. O câncer de mama é uma das neoplasias mais freqüentes na mulher, e apesar de atingir principalmente mulheres após os 50 anos, cerca de $25 \%$ dos casos ocorrem em mulheres pré-menopáusicas. Muitas dessas pacientes enfrentam uma menopausa conseqüente à quimioterapia, terapia hormonal ou procedimentos cirúrgicos.

Mais de vinte estudos procuraram avaliar o efeito da terapia hormonal ( $\mathrm{TH})$ em mulheres tratadas por câncer de mama e a recorrência do câncer mamário. A maioria absoluta desses estudos foi de delineamento observacional, não tendo sido evidenciado um maior risco de recorrência. Entretanto, o delineamento observacional pode incluir vieses. Assim, nesses estudos, as mulheres que iniciaram $\mathrm{TH}$ tinham cerca de cinco anos de intervalo livre de doença e apresentavam tumores com estadiamentos mais iniciais (13).

$\mathrm{Na}$ tentativa de produzir achados com melhores níveis de evidências, estudos prospectivos foram iniciados. Entretanto, dois estudos prospectivos abertos (HABITS Trial e Stockbolm Trial) foram interrompidos após cerca de 2,1 anos médios de seguimento por apresentarem maior taxa de recidiva no grupo de $\mathrm{TH}(\mathrm{RR}=1,8,95 \% \mathrm{CI} 1,03-3,1)$. Os dois estudos apresentaram resultados heterogêneos entre si, com significância estatística $(\mathrm{p}=0,02)$ : no HABITS, o RR foi 3,3 (95\% CI $1,5-7,4)$ e no Stockholm Trial foi de 0,82 (95\% CI 0,35-1,9). Mesmo assim, ambos os estudos se encerraram precocemente (14). Cabe comentar que há limitações no delineamento e na interpretação dos resultados desses estudos, entre elas, uma incidência $24 \%$ maior de linfonodos positivos (reconhecido como um dos principais fatores prognósticos para recorrência), e uma taxa 16\% superior de tumores receptores de estrogênio positivos no grupo de TH. Portanto, estudos de maior duração, prospectivos, com inclusão de pacientes emparelhadas pelo estadiamento entre os grupos e provavelmente em estágios mais iniciais seriam mais esclarecedores. Por outro lado, há um estudo prospectivo randomizado e duplo cego em andamento (LIBERATE Trial) com a utilização da tibolona 2,5 mg por cinco anos em mulheres tratadas por câncer de mama e com sintomas vasomotores. O término do LIBERATE Trial é previsto para o final de 2007, e seus resultados poderão trazer novas evidências sobre essa questão.

Em situações excepcionais, como frente a uma paciente tratada por câncer de mama com sinto- 
matologia climatérica severa, sem resposta satisfatória a tratamentos não hormonais, há concordância entre os autores: a $\mathrm{TH}$ poderia ser empregada desde que a paciente seja extensivamente esclarecida sobre riscos e benefícios (15).

\section{CARCINOMA DE ENDOMÉTRIO E SANGRAMENTO VAGINAL NÃO DIAGNOSTICADO}

É bem conhecido que o estrogênio estimula a mitose do tecido endometrial. A progressão anormal através de um endométrio normal para hiperplasia simples, hiperplasia complexa, atipia e carcinoma endometrial está associada à ação estrogênica sem oposição $(16,17)$, e este é o principal argumento para a contra-indicação de terapia hormonal contendo estrogênios em mulheres com história de carcinoma de endométrio, mesmo na vigência da administração associada de progestogênios.

Contudo, deve-se salientar que, em dois estudos retrospectivos que analisaram pacientes consideradas curadas após cirurgia para carcinoma endometrial em estádio I, com tumores bem diferenciados (grau I), não foi observada piora com o uso de terapia estrogênica $(18,19)$. Com base nestes trabalhos (e embora as autoras não compartilhem desta posição, no momento atual), alguns autores não consideram contra-indicação absoluta o uso de estrogênios em pacientes com baixo risco endometrial que estejam $\mathrm{cu}^{-}$ radas $(20)$, desde que sejam informadas a respeito das incertezas quanto à segurança do seu uso.

Por outro lado, a presença de sangramento vaginal em paciente na pós-menopausa deve ser sempre investigada com especial atenção em rastrear para o carcinoma de endométrio. Esta situação é considerada uma contra-indicação absoluta ao uso da terapia hormonal (18-20).

\section{PORFIRIA, DOENÇA HEPATOBILIAR E LUPUS ERITEMATOSO SISTÊMICO}

As porfirias correspondem a um grupo de doenças envolvendo síntese do grupo heme e que podem levar a manifestações agudas neuroviscerais, lesões cutâneas ou ambas (21). As porfirias hepáticas agudas são principalmente induzidas por drogas, estrogênios ou álcool, enquanto as porfirias crônicas são também causadas por hepatites virais e hemodiálise. Assim, a terapia hormonal pode desencadear as manifestações da porfiria e por isso é contra-indicada nesta situação $(22,23)$.
A presença de lupus eritematoso sistêmico (LES) corresponde a uma contra-indicação relativa à TH. Os estrogênios tradicionalmente têm sido evitados em pacientes com LES pela possibilidade de maior ativação da doença. $O$ estudo que melhor avaliou os efeitos do uso da TH em pacientes com LES foi o SELENA Trial (24). As pacientes incluídas receberam, por um ano, placebo ou estrogênio conjugados $(0,625 \mathrm{mg}$ diários $)$ associados a medroxiprogesterona ( $5 \mathrm{mg}$ por 12 dias ao mês), ambos por via oral. Foram critérios de exclusão para este estudo, pacientes com títulos elevados de anticorpo anticardiolipina, lupus anticoagulante ou com história prévia de trombose. Foi observada uma maior incidência de períodos de atividade leve a moderada, sem alteração nas taxas de atividade severa. Houve quatro eventos trombóticos no grupo da $\mathrm{TH}$ e um evento no grupo placebo. Portanto, devido a este aumento nos períodos de atividade leve a moderado no grupo ativo, o emprego de TH nas mulheres com LES deve ser considerado com cuidado, em base individual.

A doença hepática aguda é contra-indicação formal à terapia hormonal. Pacientes com disfunção hepática crônica também não deveriam receber $\mathrm{TH}$, particularmente por via oral. Entretanto, em um estudo entre pacientes com cirrose biliar primária, a TH não produziu modificações adversas ao longo de dois anos (25).

Por outro lado, alguns estudos, utilizando estrogênio por via não oral, evidenciaram ausência de efeitos negativos em pacientes com cirrose biliar primária (utilizando estradiol $50 \mathrm{mcg} /$ dia por via transdérmica associado a medroxiprogesterona $2,5 \mathrm{mg} / \mathrm{dia}$, oral) (26) ou em casos de hepatite crônica ativa (27).

\section{DOENÇA TROMBOEMBÓLICA E CARDIOVASCULAR}

O risco de tromboembolismo venoso é aumentado entre as usuárias de $\mathrm{TH}$, da mesma forma que entre usuárias de anticoncepcionais hormonais orais. Os efeitos do estrogênio no mecanismo de coagulação podem contribuir ou ser responsáveis por um estado de hipercoagulação generalizado. Os estrogênios orais afetam a síntese de fatores de coagulação por um efeito de primeira passagem hepática. $\mathrm{O}$ risco para eventos tromboembólicos com uso de $\mathrm{TH}$ é provavelmente ainda superior entre pacientes com trombofilias familiares. O uso de estrogênio ( 17 beta estradiol) por via transdérmica provavelmente se associa com um risco menor para eventos tromboembólicos em com- 
paração ao uso de estrogênio oral. Um estudo casocontrole europeu $(28,29)$ encontrou aumento de risco para episódios tromboembólicos em usuárias de $\mathrm{TH}$ oral $[\mathrm{RR}=4,2(1,5-11,6)]$, mas não entre usuárias de estrogênio por via transdérmica $[R R=0,9(0,4-2,1)]$. Entretanto, ensaios clínicos randomizados são necessários para caracterizar melhor os diferentes efeitos dos estrogênios por via não oral no risco de eventos tromboembólicos.

A prevalência de DCV em mulheres na prémenopausa é menor do que após a menopausa, quando há um acréscimo exponencial, sendo que o risco feminino se iguala ao dos homens, com a idade de 70 anos (30). Esta defasagem no período etário em que aumenta a freqüência de eventos cardiovasculares em mulheres em relação aos homens tem sido atribuída às ações do estrogênio endógeno sobre o sistema cardiovascular, por mecanismos ainda não completamente esclarecidos (31). Por outro lado, a influência da terapia hormonal ( $\mathrm{TH})$ na menopausa sobre comorbidades cardiovasculares também não está claramente estabelecida. Estudos observacionais mostraram redução de até $50 \%$ no risco para DCV em mulheres na pós-menopausa usuárias de TH (32-34).

Entretanto, os resultados de dois grandes estudos prospectivos e randomizados refutam os benefícios cardioprotetores da terapia hormonal, evidenciados nos estudos observacionais. O estudo HERS (Heart and Estrogen/Progestin Replacement Study) constituiuse num ensaio clínico randomizado para testar a eficácia da $\mathrm{TH}$ na prevenção secundária da doença cardíaca coronariana. Os resultados mostraram um aumento em eventos coronarianos no primeiro ano de seguimento após infarto agudo do miocárdio (IAM), em pacientes tratadas com uma combinação de estrogênios conjugados/acetato de medroxi-progesterona em doses fixas, com média de idade de 66,7 anos (35). Recentemente, o WHI (Women's Health Initiative), estudo prospectivo de prevenção primária da DCV, demonstrou uma incidência superior de eventos cardiovasculares em mulheres randomizadas para uso de TH similar ao estudo HERS em comparação com o grupo que recebeu placebo. Neste estudo, embora a TH tenha sido associada à redução no risco de câncer colo-retal e de fratura óssea, foi também relacionada com aumento no risco de câncer de mama (36).

Em 2004, foram publicados os dados do braço terapêutico do WHI que avaliou o uso isolado de estrogênios conjugados versus placebo em 10.739 mulheres pós-menopáusicas histerectomizadas. Houve aumento no risco de AVC ( $\mathrm{rr}=1,39$; IC: 1,10-1,77), porém uma diminuição no risco de fraturas de quadril, e efeito neutro sobre a incidência de DCV além de possível redução na incidência de câncer de mama. $\mathrm{Na}$ avaliação geral, o índice de riscos e benefícios foi neutro (37).

É importante destacar que estes estudos sofrem críticas quanto a pelo menos dois aspectos: a seleção de pacientes, que incluiu mulheres com idade, em média, 1 década superior àquela em que normalmente a TH é recomendada, e a utilização, nos 2 estudos, de um esquema de TH com drogas, via de administração e doses fixas.

Assim, embora atualmente permaneça como contra-indicação à $\mathrm{TH}$ a presença de doença cardiovascular estabelecida, as evidências indicam que o uso da TH para tratamento dos sintomas climatéricos é seguro, se iniciado no período da transição menopáusica e primeiros anos pós-menopausa $(38,39)$.

Uma reanálise recentemente publicada (40) dos dados do WHI reforçou essa hipótese, ou seja, não mostrou elevação mas, sim, uma tendência para redução de risco cardiovascular entre as mulheres que iniciaram a TH em período pós-menopáusico recente. Deve-se destacar que o estudo WHI não tinha poder estatístico suficiente para evidenciar redução de risco cardiovascular na população entre 50 e 59 anos de idade. De fato, seria necessário um número cerca de dez vezes superior de mulheres incluídas no estudo, nessa faixa etária, para observar tal efeito (41).

A hipótese de que uma intervenção mais precoce do que aquela utilizada no WHI possa exercer um efeito benéfico cardiovascular às mulheres levou ao desenho do estudo chamado KEEPS (Kronos Early Estrogen Prevention Study). Trata-se de um ensaio clínico multicêntrico, de 5 anos de duração, que avaliará a eficácia de estrogênios conjugados 0,450 $\mathrm{mg} /$ dia oral, ou $50 \mathrm{mcg}$ de estradiol transdérmico semanal (ambos associados à progesterona oral micronizada cíclica, $200 \mathrm{mg} / 12$ dias ao mês), ou placebo, em impedir a progressão da espessura medial íntima da carótida e o acúmulo do cálcio nas coronárias de mulheres entre 42 e 58 anos (todas com período pós-menopáusico máximo de 36 meses). Um total de 720 mulheres será incluído, com término previsto para 2010 (42).

Portanto, no momento atual, a TH permanece contra-indicada somente a mulheres que apresentaram eventos arteriais prévios (IAM ou AVC), ou aquelas com diversos fatores de risco cardiovascular, particularmente se pertencentes a faixas etárias mais elevadas ou apresentando período pós-menopáusico longo (mais de 10 anos). 


\section{REFERÊNCIAS}

1. WHO Scientific Group on Research on the Menopause in the 1990s. WHO technical report series 1996;866:1-107.

2. Pedro AO, Neto AMP, Paiva LH, Osis JM, Hardy E. Idade de ocorrência da menopausa natural em mulheres brasileiras: resultados de um inquérito populacional domiciliar. Cad Saúde Púb 2003:19(1):7-25.

3. Hunter MS. Emotional well-being, sexual behaviour and hormone replacement therapy. Maturitas 1990;12:299-314.

4. Belchetz PE. Hormonal treatment of postmenopausal women. N Engl J Med 1994;330:1062-71.

5. Woodruff JD, Pickar JH. Incidence of endometrial hyperplasia in postmenopausal women taking conjugated estrogens (Premarin) with medroxyprogesterone acetate or conjugated estrogens alone. Am J Obstet Gynecol 1994;170:1213-23.

6. Effects of hormone replacement therapy on endometrial histology in postmenopausal women. The Postmenopausal Estrogen/Progestin Interventions (PEPI) Trial. The Writing Group for the PEPI Trial. JAMA 1996;275:370-5.

7. Beresford SA, Weiss NS, Voigt LF, McKnight B. Risk of endometrial cancer in relation to use of oestrogen combined with cyclic progestagen therapy in postmenopausal women. Lancet 1997;349:458-61.

8. Vilodre LC, Wender MC, Castro JA, Reis FM, Ruschel S, Magalhães JA, et al. Endometrial response to cyclic regimen of percutaneous $17 \beta$-estradiol and low-dose vaginal micronized progesterone in women with mild to moderate hypertension. Gynecol Endocrinol 2003;17(4):323-8.

9. Spritzer PM, Vitola D, Vilodre LC, Wender MC, Reis FM, Ruschel S, et al. One-year follow-up of hormone replacement therapy with percutaneous estradiol and low-dose vaginal natural progesterone in women with mild to moderate hypertension. Exp Clin Endocr Diab 2003;111(5):267-73.

10. Anderson GL, Limacher M, Assaf AR, Bassford T, Beresford AS, Black $\mathrm{H}$, et al. Effects of conjugated equine estrogen in postmenopausal women with hysterectomy: the Women's Health Initiative randomized controlled trial. JAMA 2004;291:1701-12.

11. Cauley JA, Robbins J, Chen Z, Cummings SR. Effects of estrogen plus progestin on risk of fracture and bone mineral density: the Women's Health Initiative randomized trial. JAMA 2003;290:1729-38

12. Chlebowski RT, Wactawski-Wende J, Ritenbaugh C, Hubbell FA, Ascensao J, Rodabough RJ, et al. Estrogen plus progestin and colorectal cancer in postmenopausal women. N Engl J Med 2004:350:991-1004.

13. Col NF, Hirota LK, Orr RK, Erban JK, Wong JB, Lau J, et al. Hormone replacement therapy after breast cancer: a systematic review and quantitative assessment of risk. J Clin Oncol 2001:19:1357-63.

14. Holmberg L, Anderson $\mathrm{H}$; for the HABITS steering and data monitoring committees. HABITS (hormonal replacement therapy after breast cancer-is it safe?), a randomised comparison: trial stopped. Lancet 2004;363:453-5.

15. Xydakis AM, Sakkas EG, Mastorakos G. Hormone replacement therapy in breast cancer survivors. Ann N Y Acad Sci 2006; 1092:349-60.

16. Rosenwaks Z, Wentz AC, Jones GS, Urban MD, Lee PA, Migeon CJ, et al. Endometrial pathology and estrogens. Obstet Gynecol 1979;53:403-10.

17. Grady D, Gebretsadik T, Kerlikowske K, Ernster V, Petitti D. Hormone replacement therapy and endometrial cancer risk: a meta-analysis. Obstet Gynecol 1995;85:304-13.

18. Creasman WT, Henderson D, Hinshaw W, Clarke-Pearson DL. Estrogen replacement therapy in the patient treated for endometrial cancer. Obstet Gynecol 1986;67:326-30.

19. Chapman JA, DiSaia PJ, Osann K, Roth PD, Gillotte DL, Berman ML. Estrogen replacement in surgical stage I and II endometrial cancer survivors. Am J Obstet Gynecol 1996; 175:1195-200.

20. Creasman WT. Estrogen replacement therapy: Is previously treated cancer a contraindication? Obstet Gynecol $1991 ; 77: 308-12$.
21. Gross U, Hoffmann GF, Doss MO. Erythropoietic and hepatic porphyries. J Inherit Metab Dis 2000;23(7):641-61.

22. Andersson C, Innala E, Backstrom T. Acute intermittent porphyria in women: clinical expression, use and experience of exogenous sex hormones. A population-based study in northern Sweden. J Intern Med 2003;254(2):176-83.

23. Buyon JP, Petri MA, Kim MY, Kalunian KC, Grossman J. The effect of combined estrogen and progesterone hormone replacement therapy on disease activity in systemic lupus erythematosus: a randomized trial. Ann Intern Med 2005;142(12.1):953-62.

24. Bulaj ZJ, Franklin MR, Phillips JD, Miller KL, Bergonia HA, Ajioka RS, et al. Transdermal estrogen replacement therapy in postmenopausal women previously treated for porphyria cutanea tarda. J Lab Clin Med 2000;136(6):482-8.

25. Olsson R, Mattsson LA, Obrant K, Mellstrom D. Estrogenprogestogen therapy for low bone mineral density in primary biliary cirrhosis. Liver 1999;19(3):188-92.

26. Ormarsdottir $\mathrm{S}$, Mallmin $\mathrm{H}$, Naessen $\mathrm{T}$, Petren-Mallmin $\mathrm{M}$, Broome U, Hultcrantz $R$, et al. An open, randomized, controlled study of transdermal hormone replacement therapy on the rate of bone loss in primary biliary cirrhosis. $\mathbf{J}$ Intern Med 2004;256(1):63-9.

27. Rinaldi M, Cagnacci A, Pansini FE, de Aloysio D, Sgarabotto MP, Bacchi-Modena A. Neutral effect of prolonged transdermal hormone therapy on liver function of postmenopausal women with chronic active hepatitis. Menopause 2005;12(5):619-22.

28. Scarabin P, Oger E, Plu-Bureau G; on behalf of the Estrogen and ThromboEmbolism Risk (ESTHER) Study Group. Differential association of oral and transdermal oestrogen replacement therapy with venous thromboembolism risk. Lancet 2003;362:428-32.

29. Canonico M, Oger E, Plu-Bureau G, Conard J, Meyer G, Levesque $\mathrm{H}$, et al. Hormone therapy and venous thromboembolism among postmenopausal women impact of the route of estrogen administration and progestogens: the ESTHER study. Circulation 2007;115:840-5.

30. Assmann G, Carmena R, Cullen P, Fruchart JC, Jossa F, Lewis $B$, et al; for the International Task Force for the Prevention of Coronary Heart Disease. Coronary Heart Disease: reducing the risk. A worldwide view. Circulation 1999;100:1930-8.

31. Maturana MA, Irigoyen MC, Spritzer PM. Menopause, estrogens, and endothelial dysfunction: current concepts. Clinics 2007;62(1):77-86

32. Bush TL, Barret-Connor E, Cowan LD, Criqui MH, Wallace RB, Suchindran CM. Cardiovascular mortality and noncontraceptive use of estrogen in women: results from the Lipid Research Clinics Program Follow-Up Study. Circulation 1987:75:1102-9.

33. The writing group for the PEPI TRIAL. Effects of estrogen or estrogen/progestin regimens on heart disease risk factors in postmenopausal women. The postmenopausal estrogen/progentin interventions (PEPI) trial. JAMA 1995;273:199-208.

34. Grodstein F, Stampfer MJ, Colditz GA, Willet WC, Manson JE, Joffe $\mathrm{M}$, et al. Postmenopausal hormone therapy and mortality. N Engl J Med 1997;336:1769-75.

35. Hulley S, Grady D, Bush T, Fulberg C, Herrington D, Riggs B, et al. For the heart and estrogen/progestin replacement study (HERS) research group. Randomized trial of estrogen plus progestin for secondary prevention of coronary heart disease in postmenopausal women. JAMA 1998;280:605-13.

36. Rossouw JE, Anderson GL, Prentice RL, LaCroix AZ, Kooperberg C, Stefanick ML, et al; Writing Group for the Women's Health Initiative Investigators. Risks and benefits of estrogen plus progestin in healthy postmenopausal women: principal results From the Women's Health Initiative randomized controlled trial. JAMA 2002;288:321-33.

37. Anderson GL, Limacher M, Assaf AR, Bassford T, Beresford SA, Black H, et al; Women's Health Initiative Steering Committee. Effects of conjugated equine estrogen in postmenopausal women with hysterectomy. JAMA 2004:291:1701-12. 
38. Lobo RA. Evaluation of cardiovascular event rates with hormone therapy in healthy, early postmenopausal women results from 2 large clinical trials. Arch Intern Med 2004;164:482-4.

39. Salpeter SR, Walsh JME, Greyber E, Salpeter EE. Brief report: coronary heart disease events associated with hormone therapy in younger and older women. A meta-analysis. J Gen Intern Med 2006;21:363-6.

40. Rossouw JE, Prentice RL, Manson JE, Wu L, Barad D, Barnabei $V$, et al. Postmenopausal hormone therapy and risk of cardiovascular disease by age and years since menopause. JAMA 2007;297(13):1465-77.

41. Naftolin F, Taylor HS, Karas R, Brinton E, Newman J, Clarkson TB, et al. The Women's Health Initiative could not have detected cardioprotective effects of starting hormone therapy during the menopausal transition. Fertil Steril 2004;81(6):1498-501.
42. Harman SM, Brinton EA, Cedars M, Lobo R, Manson JE, Merriam GR, et al. KEEPS: The Kronos Early Estrogen Prevention Study. Climacteric 2005;8(1):3-12.

\section{Endereço para correspondência:}

Poli Mara Spritzer

Serviço de Endocrinologia

Hospital de Clínicas de Porto Alegre

Rua Ramiro Barcelos 2350

90035-003 Porto Alegre, RS

Fax: (51) 2101-8777

E-mail: spritzer@ufrgs.br 